\title{
Effects of schedule and delay of reinforcement on acquisition speed
}

\author{
E. J. CAPALDI \\ Purdue University, Lafayette, Indiana 47907
}

\begin{abstract}
The effects of schedule of reinforcement (partial vs. consistent) and delay of reward $(0$ to $20 \mathrm{sec})$ on running in rats were examined in two investigations. The effects of delay depended upon schedule of reinforcement; acquisition speed decreased as delay increased under consistent reinforcement, a common finding, while acquisition speed was independent of delay under partial reinforcement, a new finding. The partial-reinforcement acquisition effect or PRAE is defined as faster acquisition speed under partial than under consistent reinforcement. Because running speed was independent of delay under partial reinforcement, but decreased as delay increased under consistent reinforcement, the PRAE increased as delay of reinforcement increased.
\end{abstract}

The two investigations reported here were concerned with the effects of delay of reinforcement and schedule of reinforcement on acquisition speed in rats. Delay of reinforcement has been varied frequently, of course, using a schedule of consistent reinforcement, and as is well known, under consistent reinforcement acquisition speed decreases as delay increases (e.g., Campbell \& Knouse, 1972). No investigation using discrete trials seems to have been reported in which delay of reinforcement was varied using a schedule of partial reinforcement. In Experiment 1, there were two delays of reinforcement, 0 and $10 \mathrm{sec}$, and two schedules of reinforcement, consistent and 50\% irregular partial reinforcement.

With regard to schedule of reinforcement, the most frequently reported acquisition finding is that under immediate reinforcement partial and consistent groups do not differ (e.g., Theios \& McGinnis, 1967). Not infrequently, under immediate reinforcement, however, partial reinforcement has produced slower acquisition responding than consistent reinforcement (e.g., Theios, 1962). Finally, in a relatively small number of immediate reinforcement investigations (e.g., Goodrich, 1959; Wagner, 1961), partial reinforcement produced faster acquisition responding than consistent reinforcement in early runway segments, but slower responding in the goal segment. Faster acquisition responding under partial than under consistent reinforcement is called the partial-reinforcement acquisition effect or PRAE.

Only Lehr (1974) and Wilton and Clements (1973) seem to have examined partial reinforcement using

This research was supported in part by National Institute of Child Health and Development Grant HD 04379 to E. J. Capaldi. other than immediate reinforcement. Wilton and Clements, employing a 30 -sec delay, obtained a PRAE. In the Wilton and Clements investigation, delay occurred in a delay chamber anterior to the goalbox; thus a goal segment measure was not available. In two investigations by Lehr (1974), which employed 20 -sec delay delivered in the goalbox, partial and consistent reinforcement groups did not differ in acquisition. Experiment 1 here is the first to compare partial and consistent reinforcement using both immediate and delayed reinforcement.

\section{EXPERIMENT 1}

\section{Method}

Subjects. The subjects were $\mathbf{4 0}$ male albino rats, 95 days old at the start of the study, purchased from the Holtzman Co., Madison, Wisconsin.

Apparatus. The straight-alley runway, which was painted gray and was covered with $1.3-\mathrm{cm}$ hardware cloth, was $208.4 \mathrm{~cm}$ long, $10.2 \mathrm{~cm}$ wide, and had $22.9 \mathrm{~cm}$ high sides. The startbox, $20.3 \mathrm{~cm}$ long, was separated from the remainder of the alley by a guillotine door which was raised about $3 \mathrm{sec}$ after placing the rat in the startbox. Raising the guillotine door started the first of three clocks $(.01 \mathrm{sec})$. Interrupting a photobeam beyond the guillotine door stopped Clock 1 (start time) and started Clock 2. Clock 2 was stopped (run time) and Clock 3 started by interrupting a photobeam $132.1 \mathrm{~cm}$ beyond the first. Clock 3 was stopped (goal time) by interrupting a photobeam $39.4 \mathrm{~cm}$ beyond the second photobeam and $6.4 \mathrm{~cm}$ in front of a brass $5.1 \times 10.2 \times 3.8 \mathrm{~cm}$ foodcup. The brass cup contained a metal inset, $3.8 \times 3.2 \times 1.9 \mathrm{~cm}$, which held the food pellets on reinforced trials. A guillotine brass door confined the rat to the goal area. A solenoid-operated aluminum lid covered the food cup on all reinforced trials, preventing access to the pellets. This lid was recessed in the goal cup in such manner that the rat could not, while remaining on all four feet, determine prior to breaking the last photobeam if it was in the open or the closed position.

Pretraining. Upon arrival at the laboratory, the subjects were housed in individual cages with food and water freely available. 
Limited feeding, which consisted of $12 \mathrm{~g}$ /day of Wayne Lab Blox, began on Day 1 of pretraining. Water was always freely available. The subjects were divided into four groups of 10 rats each and were handled briefly on an individual basis on all pretraining days. On Days 10-12, the subjects received $1545-\mathrm{mg}$ Noyes pellets in the home cage. The number of pellets eaten either in the home cage or in the apparatus was always subtracted from the $12-\mathrm{g}$ daily ration.

Experimental Training. Experimental training began on Day 13 of deprivation. The experimental design was a 2 by 2 factorial combining two schedules of reinforcement, partial vs. consistent, and two delays of reinforcement, immediate vs. 10-sec delay of reinforcement on reinforced trials.

On nonreinforced trials, the lid of the food cup was withdrawn, exposing clearly the empty food cup. On reinforced trials the lid of the food cup was in the closed position, opening either immediately or $10 \mathrm{sec}$ after the last photobeam had been broken. Two aspects of this procedure deserve comment. First, there was no delay on nonreinforced trials, the open position of the food cup lid clearly indicating a nonreinforced trial. Second, in the terminology employed by Wilton and Clements (1973), the delayed-reinforcement PR group employed here received correlated rather than uncorrelated reinforcement. In correlated reinforcement, one cue occurs in connection with a nonreinforced trial, i.e., open food cup, another in connection with a reinforced trial, i.e., closed food cup. In uncorrelated reinforcement, cues are nondifferential with respect to nonreinforced and reinforced trials. In the Wilton and Clements investigation, correlated reinforcement produced a larger PRAE than uncorrelated reinforcement.

On nonreinforced trials, the rat was confined to the goal area for $10 \mathrm{sec}$. On reinforced trials, the rat was removed from the goalbox immediately after consuming 1545 -mg Noyes pellets. Groups CR-I and CR-D were reinforced on all trials, Group CR-I immediately on entering the goalbox and Group CR-D $10 \mathrm{sec}$ after entering the goalbox. Groups PR-I and PR-D were reinforced on half the daily trials and nonreinforced on the remaining half, reinforcement occurring immediately for Group PR-I and after a 10-sec delay for Group PR-D. There were four runway trials each day. On Days 1 through 6 , the schedules of reinforced $(t)$ and nonreinforced $(-)$ trials, repeated every 6 days were,,,,,+-+--++++---++-+--+ , -+-+ . There were 20 days of acquisition training. The rats were run in squads of eight, two from each of the four groups. The running order of the eight rats in a squad was randomized daily and all rats in a squad received trial $\mathbf{N}$ before any rat received trial $N+1$, producing an intertrial interval of about $10 \mathrm{~min}$ later in training. The rat was allowed $60 \mathrm{sec}$ to complete any runway section. If more than $60 \mathrm{sec}$ was required, the time in excess of $60 \mathrm{sec}$ was added to the time required to complete the next alley sections. If a rat failed to enter the goalbox within $180 \mathrm{sec}$, it was placed in the goalbox. After the first few days of acquisition training, a time of $60 \mathrm{sec}$ was not recorded in any alley section for any rat.

\section{Results}

Figure 1 shows running speed $(\mathrm{cm} / \mathrm{sec})$ in blocks of 2 days for each of the four groups in each of the three runway segments. In the start and run segments, Group CR-D ran more slowly than the remaining groups, which differed only slightly, and in the goal segment, Group CR-I ran faster than the remaining groups, which differed only slightly. Thus, in the start and run segments, a PRAE was obtained under delayed reinforcement but not under immediate reinforcement. And in the goal segment, while

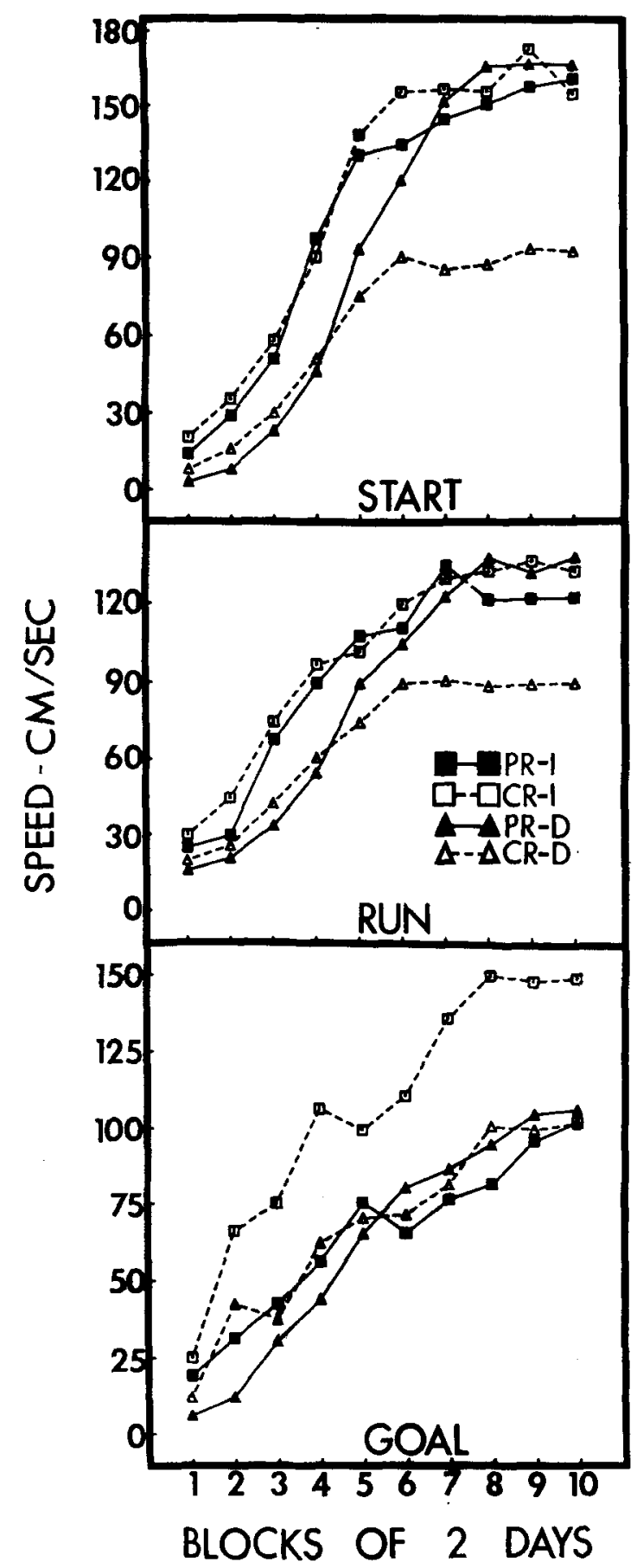

Figure 1. Running speed $(\mathrm{cm} / \mathrm{sec})$ in blocks of 2 days for each of the groups in each runway segment, stert (upper panel), run (middle panel), and goal (lower panel).

Group CR-I ran faster that Group PR-I, Groups CR-D and PR-D did not differ. Further, although speed decreased as delay of reinforcement increased under consistent reinforcement, running speed was independent of delay of reinforcement under partial reinforcement. A 2 by 2 analysis of variance applied 


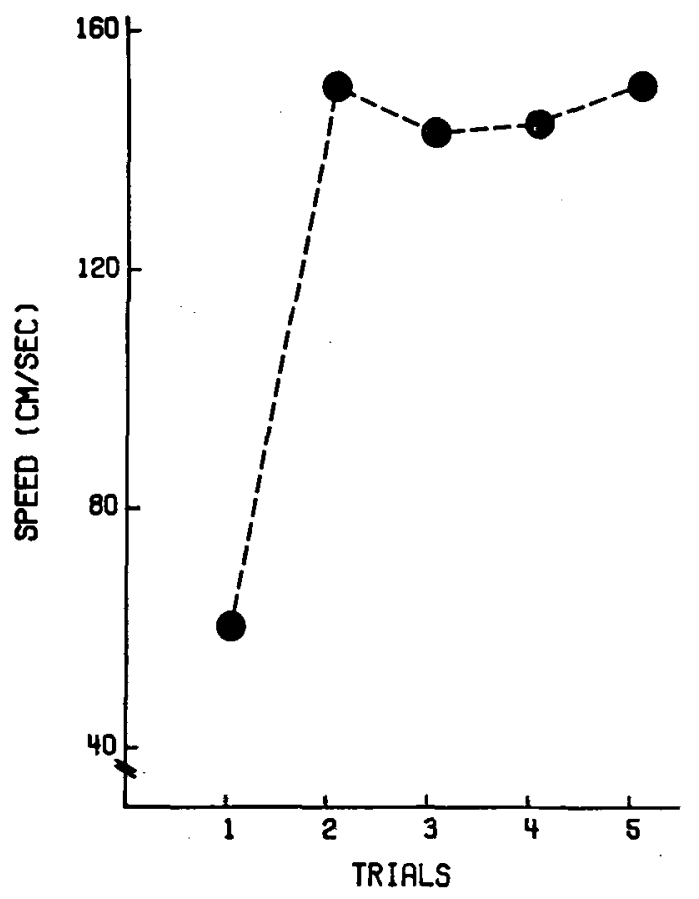

Figure 2. Running speed $(\mathrm{cm} / \mathrm{sec})$ in the run section of the apparatus on each of the five trials of the last day of acquisition for all partial reinforcement groups combined.

to the last block of acquisition trials in each alley section revealed that all differences due to schedule of reinforcement, delay of reinforcement, or the interaction of these were significant at the .01 level or better (smallest $F=7.68, p<.01$, in run for delayed reinforcement, $\mathrm{df}=1 / 36$, in all cases). Subsequent Newman-Keuls tests indicated that in the start and run segments Group CR-D differed significantly $(p<.01)$ from all other groups, which did not differ from each other, and that in the goal segment Group CR-I ran faster than all other groups $(p<.01)$, which did not differ from each other.

\section{EXPERIMENT 2}

Experiment 2 was concerned with the generality of the new relationship found in Experiment 1, that, under partial reinforcement, acquisition speed was independent of delay of reinforcement. Accordingly, Experiment 2 employed a wider range of delay values than Experiment 1: 0, 10, 15, and $20 \mathrm{sec}$, rather than merely 0 and $10 \mathrm{sec}$. In addition, Experiment 2 employed a $20 \%$ regular reinforcement schedule, the $4 \mathrm{NR}$ schedule, rather than a $50 \%$ irregular schedule. Under the 4NR schedule, there were five trials each day, the first four being nonreinforced, with only the fifth or last trial of the day being reinforced.

Also employed in Experiment 2 were consistent reinforcement groups which received $0,10,15$, or
$20 \mathrm{sec}$ delay. Experiment 2 was, then, a 2 by 4 factorial which combined two schedules of reinforcement and four delays of reinforcement.

\section{Method}

Subjects. Eighty rats were employed of the same description as those employed in Experiment 1.

Apparatus. The same runway was employed in Experiment 2 as was employed in Experiment 1.

Procedure. All aspects of the procedure employed in Experiment 2 were, except where specifically noted, identical to those employed in Experiment 1. There were 20 days of acquisition training at five trials each day. Four of the groups received consistent reinforcement in acquisition, the delay being $0 \mathrm{sec}$ (Group CR-0), $10 \mathrm{sec}$ (Group CR-10), $15 \mathrm{sec}$ (Group CR-15), or $20 \mathrm{sec}$ (Group CR-20). The remaining four groups received partial reinforcement in acquisition under a 4NR schedule, which consisted of four successive nonreinforced trials each day followed by a reinforced trial, delay on the reinforced trials being $0 \mathrm{sec}$ (Group PR-0), $10 \mathrm{sec}$ (Group PR-10), $15 \mathrm{sec}$ (Group PR-15), or $20 \mathrm{sec}$ (Group PR-20). There were 10 squads of 8 rats each, 1 rat from each group.

\section{Results}

A characteristic mode of responding, shown in Figure 2, was developed by each of the partial reinforcement groups by about Day 10 of acquisition.

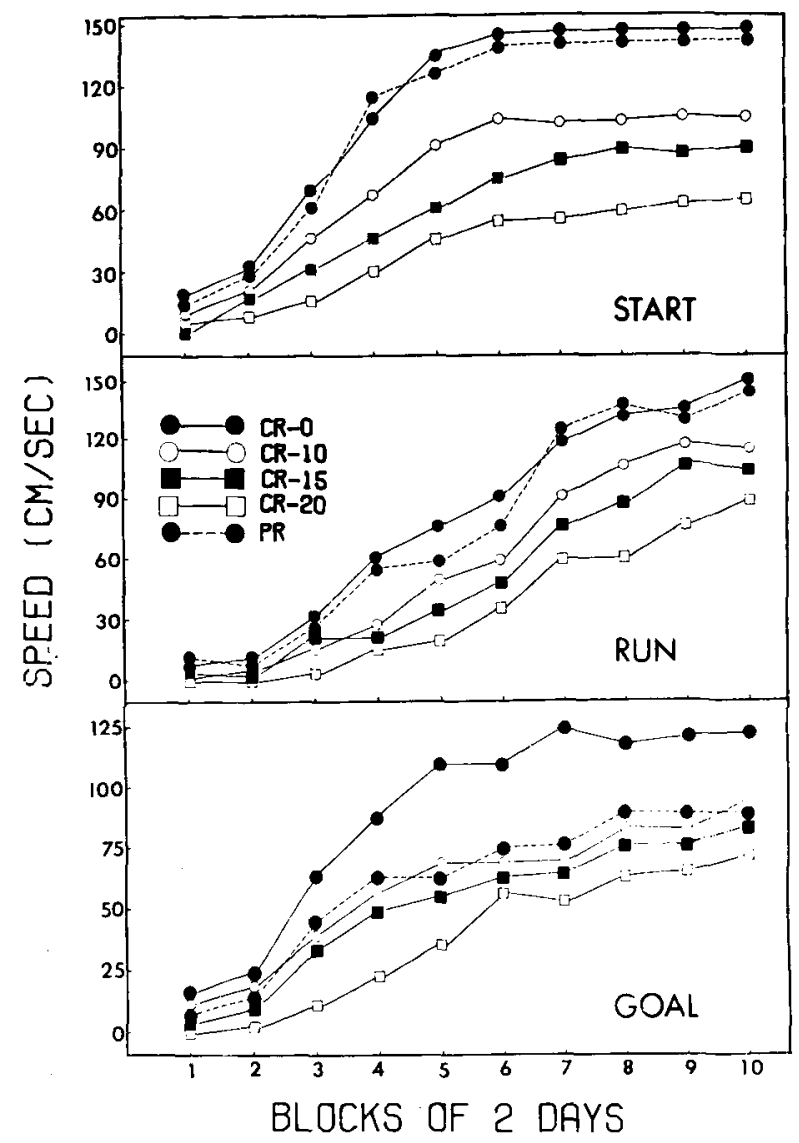

Figure 3. Running speed $(\mathrm{cm} / \mathrm{sec})$ in each of the three sections of the runway in blocks of 2 days for each of the four consistent reinforcement groups and for the four partial reinforcement groups combined. 
Figure 2 shows, for all partial reinforcement groups combined, mean running speed $(\mathrm{cm} / \mathrm{sec})$ in the run section of the apparatus on each of the five trials of the last day of acquisition. As is clear from Figure 2, running speed under the partial reinforcement 4NR schedule was slow on the first (nonreinforced) trial of the acquisition day and thereupon became rapid and nondifferential over the remaining four acquisition trials. In view of the marked difference between Trial 1 and subsequent trial performance in the partial reinforcement groups, Trial 1 data for all groups, consistent as well as partial, were eliminated from consideration here. However, including Trial 1 speeds in the daily acquisition means did not eliminate any significant difference reported here, although such inclusion reduced or augmented such differences depending upon the case.

Figure 3 shows running speed $(\mathrm{cm} / \mathrm{sec})$ in each of the three sections of the runway, in blocks of 2 days, for each of the four consistent reinforcement groups and for the four partial reinforcement groups combined. In Experiment 2, differences among the four partial reinforcement groups were for practical purposes nonexistent, thus combining the data points for these groups in Figure 3 improves graphical presentation of findings very considerably with no loss of information. For example, on the last 4 days of acquisition, mean running speed for Groups PR-0, PR-10, PR-15, and PR-20 were, respectively: in start; 140.8, 142.5, 141.9, and 143.7; in run, 136.6, 134.5, 135.9, and 134.3; and in goal, 86.5, 88.1, 85.9, and 87.1. Essentially, the findings shown in Figure 3 confirm in detail for up to 20-sec delay earlier findings reported in Experiment 1 for only 10-sec delay. Three acquisition relationships considered earlier in Experiment 1 may be mentioned in passing here. As already indicated, acquisition speed was independent of delay of reinforcement under partial reinforcement. Moreover, as shown in Figure 3, acquisition speed decreased as delay of reinforcement increased under consistent reinforcement. Finally, as shown in Figure 3, the tendency in all alley sections was for delayed partial reinforcement to produce progressively superior acquisition responding relative to delayed consistent reinforcement as delay of reinforcement increased. Under immediate reinforcement, Groúps PR-0 and CR-0 ran equally rapidly in the start and run sections, with PR-0 running more slowly than CR-0 in the goal section.

Of greatest interest statistically are the results of an analysis applied to the last 4 days of acquisition; a significant Delay of Reinforcement by Schedule of Reinforcement interaction was obtained in each alley section $[F(3,72)=6.38, p<.01$, in start; 5.27, $\mathrm{p}<.01$, in run; and 5.15, $\mathrm{p}<.01$, in goal]. This interaction reflects the tendency for running speed to decrease with increasing delay under consistent reinforcement while being independent of delay of reinforcement under partial reinforcement. Newman-Keuls tests indicated that the four partial reinforcement groups did not differ in any alley section. Also, Newman-Keuls tests indicated that there was only one difference among the consistent reinforcement groups which failed to reach a .05 level of statistical significance in at least one alley section, that between Groups CR-10 and CR-15.

\section{Discussion}

In Experiment 2, as in Experiment 1 and in many other previous investigations, acquisition speed decreased as delay of reinforcement increased under consistent reinforcement. But in Experiment 2, under partial reinforcement, acquisition speed was independent of delay of reinforcement in the range from 0 to $20 \mathrm{sec}$, a relation obtained in Experiment 1 but only for delays of 0 and $10 \mathrm{sec}$. In Experiment 2, a PRAE was not shown by the immediate reinforcement groups, but was shown by all delay groups. This finding is also consistent with the results of Experiment 1, in which a PRAE occurred under a 10-sec delay of reinforcement but not under immediate reinforcement.

The results of Experiments 1 and 2 suggest that a PRAE is more likely the longer the delay of reinforcement. This is because, although under consistent reinforcement acquisition speed declined as delay of reinforcement increased, acquisition speed was independent of delay of reinforcement under partial reinforcement. Indirect evidence available from previous investigations is consistent with the proposition that a PRAE is more likely under delayed than under immediate reinforcement. For example, as indicated earlier, a PRAE is seldom obtained under immediate reinforcement, and even where it is obtained in the early alley segments, running associated with partial reinforcement is slower than that associated with consistent reinforcement in the goal or terminal runway segment. In contrast, Wilton and Clements (1973) obtained a PRAE using delayed reinforcement, and in two investigations reported by Lehr (1974), while delayed reinforcement did not produce a PRAE, neither did delayed partial reinforcement produce slower running than delayed consistent reinforcement in any alley segment including the goal segment. Thus, considering all available evidence, not only has the PRAE occurred with some regularity under delayed reinforcement, but delayed partial reinforcement has yet to produce slower running than delayed consistent reinforcement in any alley segment. 


\section{GENERAL DISCUSSION}

In previous investigations, the relationship between acquisition responding and delay of reinforcement was examined using consistent reinforcement. Under consistent reinforcement, acquisition speed tends to decrease as delay of reinforcement increases. Accordingly, it has come to be generally accepted that acquisition speed tends to decrease as delay of reinforcement increases (see, e.g., Campbell \& Knouse, 1972). In the two investigations reported here, while acquisition speed decreased as delay of reinforcement increased under consistent reinforcement, acquisition speed was independent of delay under partial reinforcement. However, the maximum delay employed here was $20 \mathrm{sec}$, and it would plainly be improper to assume at this point that acquisition speed will remain independent of delay of reinforcement for delays longer than $20 \mathrm{sec}$. Nevertheless, and this should be emphasized, the partial reinforcement findings obtained here do suggest that consistent reinforcement provides less than a fully adequate representation of the relationship between acquisition responding and delay of reinforcement.

What can be said safely about the relationship between acquisition speed and delay of reinforcement on the basis of the present findings is that the tendency for acquisition speed to decrease as delay increased is greater under consistent reinforcement than under partial reinforcement. In order to arrive at a more general conclusion, additional research is required. For example, if the tendency, or lack of it, for acquisition speed to decrease as delay increases turns out to be quite sensitive to percentage of reinforcement, then it may be necessary to examine simultaneously a wide variety of reinforcement percentages and a wide variety of delay values. Whatever may turn out to be the case, however, the findings obtained here alert us to the fact that in order to understand properly the relationship between acquisition responding and delay of reinforcement, a much wider range of experimental conditions will have to be employed than have been utilized in the past.

Turning to explanation, it may be indicated that the present experiments did stem from a particular theory or approach which they support, the reinforcement level view (Capaldi, 1974, in press; Capaldi \& Haggbloom, 1974). Theoretical comments will be restricted to a brief few from the reinforcement level approach. Such restriction seems necessary because far more than a manageable number of models could be applied to the present findings, both of delay of reinforcement acquisition models and partial reinforcement models. Too, application of any of these models to the present findings would involve substantial guesswork because, with the exception of the Wilton and Clements (1973) information model, none has directly considered the effects of delayed partial reinforcement on acquisition responding. Offhand, it would seem that many models would predict that, with increasing delay acquisition, speed should decrease similarly for partial and consistent reinforcement, but that remains to be seen.

The reinforcement level view suggests that the results obtained here may be due in substantial part to inhibitory growth which occurs in the delay interval prior to the delivery of reinforcement. Such inhibitory growth is seen as being greater, the longer the delay. However, differences in such inhibitory growth should be smaller, the lower the percentage of reinforcement. Since this is so, the reinforcement level view suggests that the lower the percentage of reinforcement, the smaller should be performance differences due to increasing delay.

\section{REFERENCES}

Campbell, P.E. \& Knouse S.B. Extinction following delayed reward: A review. Psychonomic Monograph Supplements, 1972, 4(15, Whole No. 63).

Capaldi E.J. Partial reward either following or preceding consistent reward: A case of reinforcement level. Journal of Experimental Psychology, 1974, 102, 954-962.

Capaldi, E.J. Reinforcement level: An expectancy-associative approach to relative reinforcement and nonreinforcement effects. In G. McCain (Ed.), The Arlington Symposium on learning. Stamford, Connecticut: Greylock, in press.

Capaldi E.J., \& Haggbloom, S.J. Influence of reward magnitude on the initial nonreward effect. Bulletin of the Psychonomic Society, 1974, 3, 219-221.

Goodrich, K.P. Performance in different segments of an instrumental response chain as a function of reinforcement schedule. Journal of Experimental Psychology, 1959, 59, 57-63.

Lehr, R. Partial reward and positive contrast effects. Animal Learning \& Behavior, 1974, 3, 221-224.

Theios, J. The partial reinforcement effect sustained through blocks of continuous reinforcement. Journal of Experimental Psychology, 1962, 64, 1-6.

Theios, J. \& McGinnis, R.W. Partial reinforcement before and after continuous reinforcement. Journal of Experimental Psychology, 1967, 73, 479-481.

Wagner, A.R. Effects of amount and percentage of reinforcement and number of acquisition trials on conditioning and extinction. Journal of Experimental Psychology, 1961, 63, 234-242.

Wilton, R.N., \& Clements, R.O. Information and the partial reinforcement acquisition effect. Quarterly Journal of Experimental Psychology, 1973, 25, 147-153.

(Received for publication September 27, 1977; revision accepted January 4, 1978.) 